\title{
SLIDING MODE BASED INVERSE MODEL CONTROL FOR OIL TANKER GUIDANCE
}

\author{
Meghan Loo, Euan W. McGookin and David J. Murray-Smith \\ Department of Electronics \& Electrical Engineering, University of Glasgow, \\ Glasgow, G12 8LT, U.K. Tel: +44 1413306137 \\ Email:m.loo@elec.gla.ac.uk
}

\begin{abstract}
An inverse model based controller application is presented in this paper. In this application, inverse model control is implemented as a feedforward controller with assistance from a feedback controller. A comparison of performance is deduced between the proposed combined control structure, and that of a conventional feedback controller on its own. The corrective and feedback controller are designed using sliding mode control theory in order to regulate the heading dynamic for a nonlinear tanker model. In order to provide a more realistic evaluation, wave disturbances are implemented to provide a better comparison for the proposed control structure. Copyright (C) 2005 IFAC
\end{abstract}

Keywords: Ship control; Inverse dynamics control; Sliding mode control

\section{INTRODUCTION}

Super-tanker accidents and associated oil spills come at a high cost in terms of environmental damage and clean-up operations. Many of the problems experienced in the past decade with large oil tankers relate to navigational safety and are associated with the increasing number and size of these vessels.

Although navigational and guidance issues can be identified as a common factor in many accidents, improvements are not easily achieved because of the limited operational range of the rudder, which is the primary actuator for ship steering control in vessels of this kind. Large rudder deflections may be needed to produce rapid and large changes in the ship's heading trajectory (Fossen, 1994) and such deflections may take the rudder to the limits of its operating envelope. In such circumstances there is no control authority to deal with further unexpected navigational demands or to compensate for external disturbances, resulting in the vessel becoming temporarily uncontrollable.

In recent years considerable interest has been shown in the development of guidance systems that reduce the risk of extreme scenarios of the type outlined above (Kallstrom et al., 1979; Fossen, 1994). This paper describes an approach to guidance/navigation system design that involves the use of inverse-model based feedforward control in conjunction with corrective feedback control using sliding mode principles. Ideally, an inverse model should be capable of providing all the needed control action without the need for feedback (Magni and Terlouw, 1997). However, perfect control through model inversion is possible only if the ship model is known exactly and no external disturbances are acting on the vessel. Neither of these requirements can be regarded as practical since no mathematical model of a real system is ever perfect and ships are inevitably subject to externally generated forces through the action of tidal and other currents, wind disturbances and waves. Thus, for this type of application corrective feedback control action is inevitably required in conjunction with any form of feedforward control. The corrective controller is required to provide the control action that is not provided by the feedforward controller to compensate for external disturbances and model uncertainties (Magni and Terlouw, 1997).

The general and well-established feedback control methodology used in this investigation is sliding mode control (Healey and Marco, 1992; Edwards and Spurgeon, 1998; McGookin et al., 2000). A nonlinear oil tanker model (Fossen, 1994) has been used to evaluate the performance of the proposed system and wave disturbances are implemented within the system simulation to investigate the disturbance rejection capabilities of this combined feedforward and feedback controller structure.

\section{TANKER MODEL}

The tanker investigated is a $190000 \mathrm{dwt}$ oil tanker for which a nonlinear mathematical model is available. This model has been used in previous studies by a number of other investigators (Kallstrom et al., 1979; Fossen, 1994; McGookin et al., 2000). This model can be represented in the following standard state space form: 


$$
\dot{x}=f(x, u) \text {. }
$$

Assuming all the states are available, here $\boldsymbol{x}$ represents the system state vector and $\boldsymbol{u}$ is the input vector to the tanker. The dynamics (defined about the body-fixed inertial reference frame) and the kinematic states (defined about the earth-fixed inertial reference frame) for this model are defined in Fig 1 and Table 1 (Fossen, 1994) shown below:

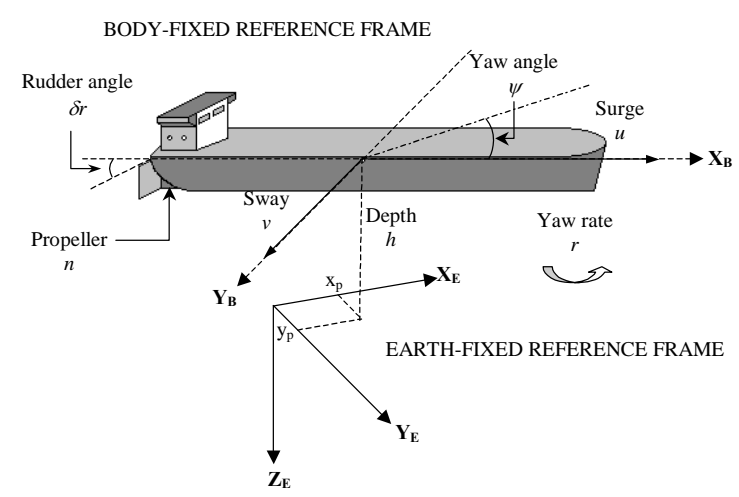

Fig 1. Tanker Reference Frames

Table 1 State and input definition

\begin{tabular}{lll}
\hline \multicolumn{2}{l}{ Body fixed states (kinetic) } & Units \\
\hline$u$ & (surge velocity) & $\mathrm{m} / \mathrm{s}$ \\
$v$ & (sway velocity) & $\mathrm{m} / \mathrm{s}$ \\
$r$ & (yaw/heading rate) & $\mathrm{rad} / \mathrm{s}$ \\
$\delta r$ & (actual rudder deflection) & $\mathrm{rad}$ \\
$n$ & (actual propeller speed) & $\mathrm{rps}$ \\
\hline \multicolumn{2}{l}{ Earth fixed states (kinematic) } \\
\hline$x_{\text {pos }} \quad$ (x position coordinate) & $\mathrm{m}$ \\
$y_{p o s} \quad$ (y position coordinate) & $\mathrm{m}$ \\
$\psi$ & (yaw/heading angle) & $\mathrm{rad}$ \\
\hline Commanded inputs & \\
\hline$\delta r_{c}$ & (commanded rudder deflection) & $\mathrm{rad}$ \\
$n_{c}$ & (commanded propeller speed) & $\mathrm{rps}$ \\
$h$ & (water depth) & $\mathrm{m}$
\end{tabular}

From the table above, it should be noted that water depth, $h$, influences the model dynamics. In this investigation, it is assumed that the vessel is travelling in deep water (with water depth $200 \mathrm{~m}$ ) so that water depth effects have negligible influence on the yawing motion and surge velocity of the vessel. It can be noted that there is a distinction between commanded inputs and the actual state vector of rudder deflection, $\delta r$, and propeller speed, $n$. This imposes a maximum actuator rate of $\dot{\delta}_{\max }=2.33^{\circ} / \mathrm{sec}$ and maximum amplitude limits $\delta r_{\max }=30^{\circ}$ and $n_{\max }=80 \mathrm{rpm}$ on each of the inputs. These limits ensure that the vessel is performing within the normal operational envelope for the rudder and propeller.

\section{WAVE DISTURBANCE}

System robustness to the effects of environmental disturbances can be investigated using wave disturbances within the computer-based model of the vessel and the associated controller. Wind generated waves are considered, with the associated forces and moments induced by a regular sea on a block-shaped ship (Fossen, 1994) The resulting forces act in the direction of the $X, Y$ forces and the $N$ moment are $X_{\text {wave }}, Y_{\text {wave }}$, and $N_{\text {wave }}$ respectively. These wave force components form a vector called $\tau_{W}$, and by applying the principle of linear superposition (Fossen, 1994), the wave forces and moments are added to the statespace equation shown in Equation (1), $\dot{\boldsymbol{x}}=\boldsymbol{f}\left(\boldsymbol{x}, \boldsymbol{u}, \tau_{W}\right)$. The vector, $\tau_{W}$, consists of three components, namely:

$$
\tau_{W}=\left[\begin{array}{c}
\sum_{i=1}^{N} \rho g B L T \cos (\beta-\psi)\left[A_{i} k_{i} \sin \left(w_{e i}+\phi_{i}\right)\right] \\
\sum_{i=1}^{N}-\rho g B L T \sin (\beta-\psi)\left[A_{i} k_{i} \sin \left(w_{e i}+\phi_{i}\right)\right] \\
\sum_{i=1}^{N} \frac{1}{24} \rho g B L\left(L^{2}-B^{2}\right) \sin 2(\beta-\psi)\left[A_{i} k_{i} \sin \left(w_{e i}+\phi_{i}\right)\right]
\end{array}\right]
$$

Here $B, L$ and $T$ are the breadth (m), length (m) and draft $(\mathrm{m})$ respectively, of the wetted part of the vessel, represented as a rectangular cuboid. The term $\rho$ is the density of water $\left(\mathrm{kg} / \mathrm{m}^{3}\right), g$ is the acceleration due to gravity $\left(\mathrm{m} / \mathrm{s}^{2}\right)$ and $(\beta-\psi)$ is the angle between the heading of the ship and the direction of the wave (rad). In addition, $\omega_{e i}$ is the frequency of encounter corresponding to wave component, $i$ and $\phi_{i}$ is a random phase angle, uniformly distributed. $A_{i}$ is the wave amplitude, $\left(A_{i}^{2}=2 S\left(w_{i}\right) \Delta w\right)$ and $k_{i}$ is the wave number, $\left(k_{i}=w_{i}^{2} / g\right)$ with $\omega_{i}$ is the wave frequency of wave component, and $\Delta \omega$ is a constant difference between successive frequencies.

In this paper, the Pierson-Moskowitz (PM) Wave Spectrum is chosen as it represents wave spectra of a fully developed sea of the North Atlantic Ocean (Fossen, 1994). The PM spectrum is written as:

$$
S(\omega)=0.0081 g^{2} \omega^{-5} \exp \left(-3.11 \omega^{-4} / H_{s}^{2}\right)
$$

Here $H_{s}$ is the significant wave height $(\mathrm{m})$. In this application, the significant wave height of $5 \mathrm{~m}$ is chosen, which corresponds to very rough sea conditions.

\section{CONTROL THEORIES}

\subsection{Linearised formulation}

The inverse model structure considered here is based on the linearised form of Equation (1), which can be represented by a general linear state space equation:

$$
\dot{\boldsymbol{x}}=\boldsymbol{A x}+\boldsymbol{B u}
$$

Here $\boldsymbol{A}$ and $\boldsymbol{B}$ are the system and control input matrices, respectively. By rearranging Equation (4), the following inverse control law can be derived:

$$
u=B^{-1}(\dot{x}-A x)
$$

Unfortunately, the number of inputs is less than the number of actual states, hence the $\boldsymbol{B}$ matrix is a nonsquare matrix. Consequently a direct matrix inversion cannot be applied in this case. An alternative approach to inverting the $\boldsymbol{B}$ matrix involves applying Singular Value Decomposition (SVD) to compute a 'pseudo-inverse', denoted by $\boldsymbol{B}^{+}$(Skogestad and Postlethwaite, 1996). This provides a suitable approximation to the inverse of $\boldsymbol{B}$. 


\subsection{Inverse Model Structure}

As mentioned previously, perfect inversion cannot be achieved. Therefore the inverse model control in Equation (5) is implemented as a feedforward controller with assistance from a feedback controller (see Fig 2).

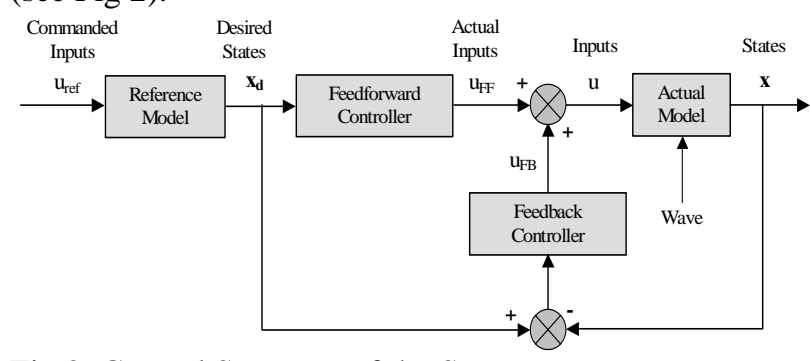

Fig 2. Control Structure of the System.

Even though, the feedforward controller is unable to provide all the control action within the system, it exhibits an "inherent robustness" compared with a pure feedback system. A feedback controller is introduced into the system to manage the remaining control action that is not provided by the feedforward controller, thus providing better trajectory following. It is commonly known as 'corrective controller' because of the nature and purpose of its control action.

As shown in Fig 2, the reference model generates desired state trajectories or responses, $\boldsymbol{x}_{\boldsymbol{d}}$, which are also known as desired states. The inverse model controller, as the feedforward controller, uses this information to generate an output signal, $u_{F F}$, which is combined with the feedback signal from the corrective controller, $u_{F B}$, to form the control input to the ship $\left(u=u_{F F}+u_{F B}\right)$. The corrective controller determines the corrective feedback signal by using the measurement error signal between the actual and desired states. This ensures that the actual state variables follow the desired states.

In this application the tanker system is decoupled into two subsystems, namely heading $\left(\boldsymbol{x}_{\boldsymbol{H}}=[v, r, \psi]^{T}\right)$ and propulsion $\left(x_{P}=[u, n]^{T}\right)$ which are the only controllable dynamic subsystems that are considered here. Each subsystem is controlled separately.

\subsection{Sliding Mode Control Theory}

In this paper, Sliding Mode (SM) control theory is used to design the feedback and corrective controllers for the heading and propulsion subsystems (Edwards and Spurgeon, 1998; McGookin et al. 2000). The advantage of using SM controller in this application is that it can provide inherent robustness to compensate for uncertainties caused by unmodelled dynamics and/or external disturbances (McGookin et al., 2000).

In this application, the design of the SM controller is based a single-input-single-output (SISO) linear representation of the separate heading and propulsion dynamics for the models (Healey and Marco, 1992).
This controller regulates the heading angle $(\psi)$ of the vessel by providing the required rudder deflection, $\delta r_{C}$ signal and propulsion $(u)$ dynamics by providing the required propeller velocity, $n_{C}$ signal. The SM control action regulates the measurement error between the desired and actual states. Using this information, the SM controller generates the required control action for accurate tracking. The SM control methodology used here is based on a SISO state-space representation of the dynamics of the vessels, similar to Equation (4). This is obtained by decoupling the heading and propulsion subsystems from the full tanker model.

All the control action provided by SM controller is through the single input, $u_{F B}$, of the system. By definition (Edwards and Spurgeon, 1998; McGookin et al., 2000), such control action of SM control has two distinct elements:

$$
u_{F B}=u_{e q}+u_{s w}
$$

where $u_{e q}$ is called the equivalent control signal and $u_{s w}$ is called the switching control signal.

The nominal equivalent control component is chosen as a state feedback gain controller, which is based on a linearised controller. It can be represented by:

$$
u_{e q}=-k^{T} x
$$

Here $k^{T}$ is the transpose of the feedback gain matrix, $k$. This is achieved through robust eigenstructure assignment (McGookin et al., 2000).

The additional control is provided by the nonlinear switching term is derived from a state hyperplane called the sliding surface, $\sigma$ (Healey and Marco, 1992; Fossen, 1994; McGookin et al., 2000) and can be represented by the following equation:

$$
\sigma(\Delta x)=h^{T} \Delta x=h^{T}\left(x_{d}-x\right)
$$

This is a function of the state error $\Delta x$ and a gain vector $h$. Following the derivation given by McGookin, et al. (2000), the switching component can be represented by:

$$
u_{s w}=\left(h^{T} b\right)^{-1}\left[h^{T} \dot{x}_{d}-\eta \operatorname{sgn}(\dot{\sigma}(\Delta x))\right]
$$

The switching term provides the nonlinear action essential for SM control and, as shown in Equation (9), this is conventionally based on the discontinuous signum function with $\eta$ as the switching gain (Edwards and Spurgeon, 1998; McGookin et al., 2000). In the paper by Healey and Marco (1992), this hard switching is replaced by a hyperbolic tangent of a continuous function. The tanh function has the same end points as the signum function (i.e., \pm 1 as $\sigma \rightarrow \infty$ ) but the boundary layer $\sigma$ has a gradual transition towards zero and the boundary layer thickness, $\phi$, determines the slope of the transition. When $\phi$ is small the transition from -1 to 1 is fast, but as $\phi$ increases the transition becomes less rapid. The switching control action with the hyperbolic tangent term included can be written as:

$$
u_{s w}=\left(h^{T} b\right)^{-1}\left[h^{T} \dot{x}_{d}-\eta \tanh (\sigma(\Delta x) / \phi)\right]
$$


The introduction of the boundary layer reduces the risk of chattering which is a feature of conventional forms of SM involving the signum function. By combining Equation (7) and (10), the full SM control structure can be represented by:

$$
u_{F B}=-k^{T} x+\left(h^{T} b\right)^{-1}\left[h^{T} \dot{x}_{d}-\eta \tanh (\sigma(\Delta x) / \phi)\right]
$$

Even though SM controller is able to offer the robustness to achieve satisfactory tracking precision, it might not achieve minimum rudder deflection. In this application accurate tracking is the first priority. As a result, the SM controller has to be tuned for high tracking accuracy but low levels of rudder deflection are also required (McGookin et al., 2000).

\subsection{Total Control Expression}

The combined control efforts from the feedforward controller shown in Equation (5) and the corrective feedback controller shown in Equation (11) form the total control input to the ship, which can be written as: $\boldsymbol{u}=\boldsymbol{B}^{+}\left(\dot{x}_{d}-A x_{d}\right)+\left\{-\boldsymbol{k}^{T} x+\left(h^{T} b\right)^{-1}\left[h^{T} \dot{x}_{d}-\eta \tanh (\sigma(\Delta x) / \phi)\right]\right\}$ This combined control signals ensures accurate tracking for the decoupled heading and propulsion subsystems.

\section{PERFORMANCE EVALUATION}

The following section evaluates the performance of sliding mode controller operating as a pure feedback controller and as a corrective controller with inverse model control as feedforward control. The controller parameter values are tuned manually for each scenario for a critically damped second order response for heading manoeuvre of $45^{\circ}$ and a surge velocity of $8 \mathrm{~m} / \mathrm{s}$ reduced to $7 \mathrm{~m} / \mathrm{s}$ for propulsion control. In each of the diagrams follow this section, the dashed line represents the desired response for the corresponding states, and the solid line represents the actual states. The time history responses for the heading angle $(\psi)$, heading error $\left(\Delta \psi=\psi_{d}-\psi\right)$, rudder deflection $(\delta r)$, surge velocity $(u)$, surge error $\left(\Delta u=u_{d}-u\right)$ and shaft velocity $(n)$ are shown in each figure. The first two parameters to be chosen for heading subsystem are the pole positions for the closed loop system. These are used to determine the state feedback vector, $k$, of the equivalent controller. For the heading dynamics, there should be three poles for the three states, but only two are tuned because the pole that is associated with heading angle feedback is set to zero as a result providing an integral action within the controller structure. Consequently, only one closed loop pole is tuned for the propulsion dynamics. The remaining two parameters are from the nonlinear switching part of the controller. Theses are switching gain, $\eta$, which determines the amplitude of the switching action, and the boundary layer thickness, $\phi$, which determines the region of gradual transition for chatter removal.

\subsection{Sliding Mode Control as feedback controller.}

In this section, sliding mode control methodology is applied as a pure feedback controller for the decoupled heading and propulsion subsystems i.e. no inverse model control. The manually tuned parameter values for each subsystem are shown in Table 2 with the corresponding simulated result shown in Fig 3.

Table 2. Design parameter values for SM controller as a pure feedback controller

\begin{tabular}{ccc}
\hline Parameter values & Heading & Propulsion \\
\hline $1^{\text {st }}$ Closed-loop pole & $\mathrm{ph} 1=-0.029$ & $\mathrm{pp} 1=-1.235$ \\
$2^{\text {nd }}$ Closed-loop pole & $\mathrm{ph} 2=-0.080$ & N/A \\
Switching Gain & $\eta_{\mathrm{H}}=1.2$ & $\eta_{\mathrm{P}}=0.41$ \\
Boundary Layer & $\phi_{\mathrm{H}}=0.31$ & $\phi_{\mathrm{P}}=0.81$ \\
Thickness & &
\end{tabular}
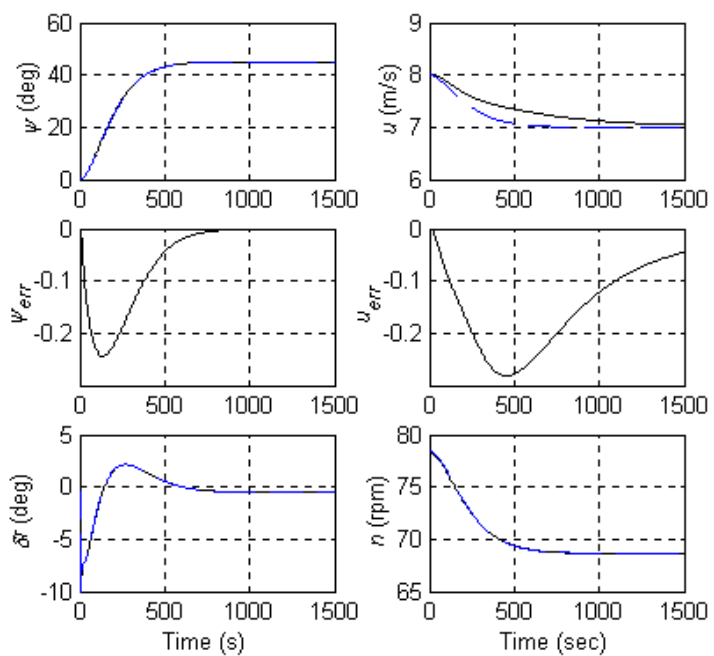

Fig. 3. Responses for SM controller as pure feedback controller without wave disturbance.

It can be clearly seen that the SM controller regulates the course heading and propulsion of the tanker to track the desired trajectory with great accuracy $(\Delta \psi=$ $-0.25^{\circ}$ and $\Delta u=-0.3 \mathrm{~m} / \mathrm{s}$, respectively). Therefore, the tracking accuracy for both subsystems is considered to be satisfactory. The initial spike observed in the rudder deflection time history is due to the initial steering of the tanker towards the desired heading trajectory.

\subsection{Sliding Mode Control as corrective controller with inverse model control as the feedforward controller.}

In this section, the main objective is to evaluate the performance of an inverse model controller working in conjunction with a SM type of corrective controller. Parameter values for the SM controller are shown in Table 2 and the corresponding result for the combined control action is shown in Fig 4.

In Fig 4, inverse model control is implemented as the feedforward controller, it can be seen that for the heading subsystem the SM control action reduces the tracking error very effectively $\left( \pm 0.3^{\circ}\right)$. However, for the propulsion subsystem, the SM controller is unable to track the desired surge velocity and shaft velocity. This is because, for the propulsion subsystem, only 
one closed-loop pole is used to tune the SM controller and slight changes of this pole can affect the responses. As a result further fine-tuning is required for the propulsion subsystem.
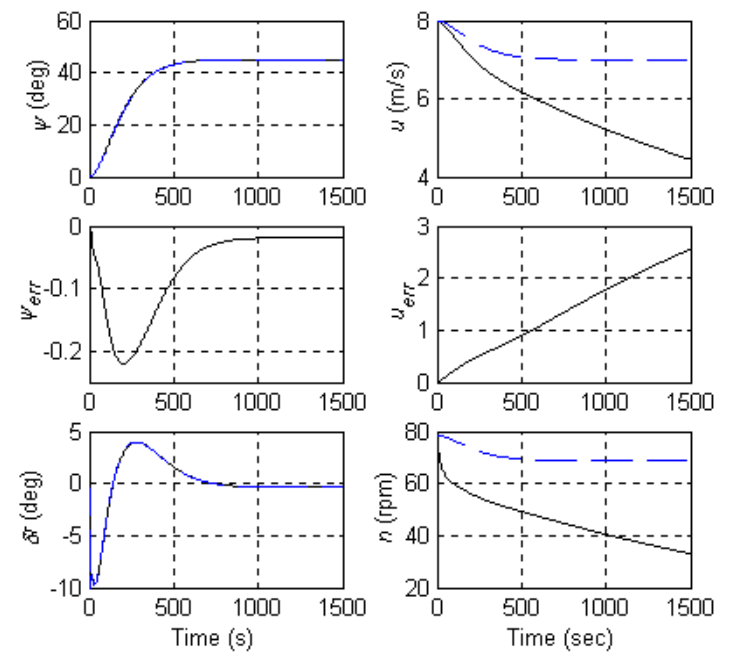

Fig. 4. Responses for inverse model controller with SM controller as the corrective controller with parameter values (from Table 2) before retuning.

The parameter values for the heading and propulsion subsystems required retuning to achieve satisfactory responses. New parameter values obtained from this retuning procedure are shown in the table below:

Table 3. Design parameter values for SM controller as a corrective controller

\begin{tabular}{ccc}
\hline Parameter values & Heading & Propulsion \\
\hline $1^{\text {st }}$ Closed-loop pole & ph1 $=-0.026$ & $\mathrm{pp} 1=-1.215$ \\
$2^{\text {nd }}$ Closed-loop pole & $\mathrm{ph} 2=-0.045$ & N/A \\
Switching Gain & $\eta_{\mathrm{H}}=1.10$ & $\eta_{\mathrm{P}}=0.35$ \\
Boundary Layer & $\phi_{\mathrm{H}}=0.30$ & $\phi_{\mathrm{P}}=0.70$ \\
Thickness & & \\
\hline
\end{tabular}
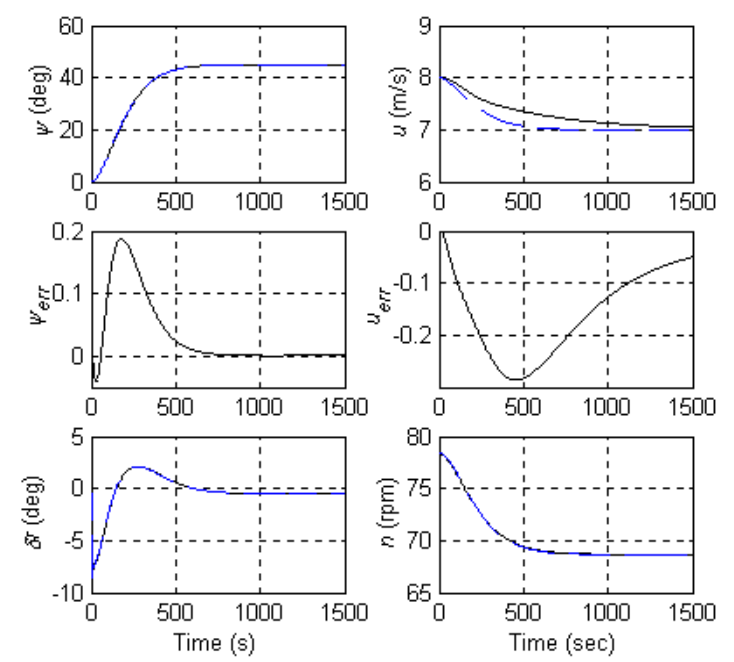

Fig 5. Results of Sliding Mode control as corrective controller with tuned parameters.

Comparing of the parameter values in Table 3 with those in Table 2 show that gains are lower for the combined controller than for the of SM controller on its own without any feedforward action. This is because inverse model control provides a nominal control signal to the system and SM provides the remaining control action. Therefore the inverse model controller reduces the task undertaken by the feedback controller.

With the inverse model controller implemented, the tracking ability of the heading subsystem shown in Figure 5 shows that it is able to offer slightly better heading accuracy $\left( \pm 0.2^{\circ}\right)$ compare with that of the SM controller on its own. On the other hand no improvement was observable for the propulsion subsystem performance. Therefore from the results obtained in Fig 4 and 5, the inverse model controller working in conjunction with the SM feedback controller offers a slight improvement in tracking ability but with lower values of gain.

\subsection{Sliding Mode Control as feedback controller in the presence of wave disturbance.}

In order to evaluate the robustness of the controllers, wave disturbances were introduced. A significant wave height of $5 \mathrm{~m}$ was chosen, corresponding to a very rough sea condition with wind speeds up to 30 knots.
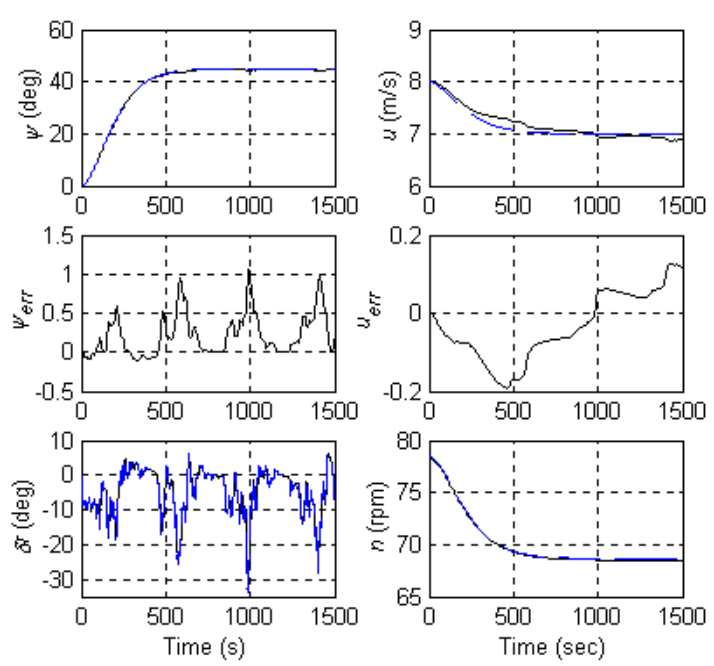

Fig. 6. Sliding mode as pure feedback controller in the presence of wave disturbance

Firstly, the performance of a SM controller as a pure feedback controller is investigated. The parameter values chosen in this case are the same as shown in Table 2. The simulated result obtained in the presence of wave disturbances is shown in Fig. 6.

As in the case for the SM controller for the heading subsystem, the heading and heading error plots show clearly that the SM controller accurately regulates the course heading of the tanker with $\Delta \psi=1^{\circ}$. However, by observing the rudder deflection at simulation times around 1000 seconds it may seen that the rudder deflection has reached $30^{\circ}$, which is the maximum rudder deflection. This is not an ideal case, as it 
saturates the rudder, thus not allowing any further control manoeuvrability for the vessel.

In the case for the propulsion subsystem, the SM controller is able to track the desired trajectories with good accuracy of $\Delta u= \pm 0.2 \mathrm{~m} / \mathrm{s}$. In addition, little distinction can be made between the commanded (dashed line) and actual shaft velocity (solid line) responses. This suggests that the tracking accuracy of the propulsion subsystem is satisfactory.

\subsection{Sliding Mode Control as corrective controller with inverse model control as the feedforward controller in the presence of wave disturbance.}

In this section, the performance of an inverse model controller working in conjunction with a SM controller as the corrective controller is investigated. The parameter values used are obtained from Table 3.
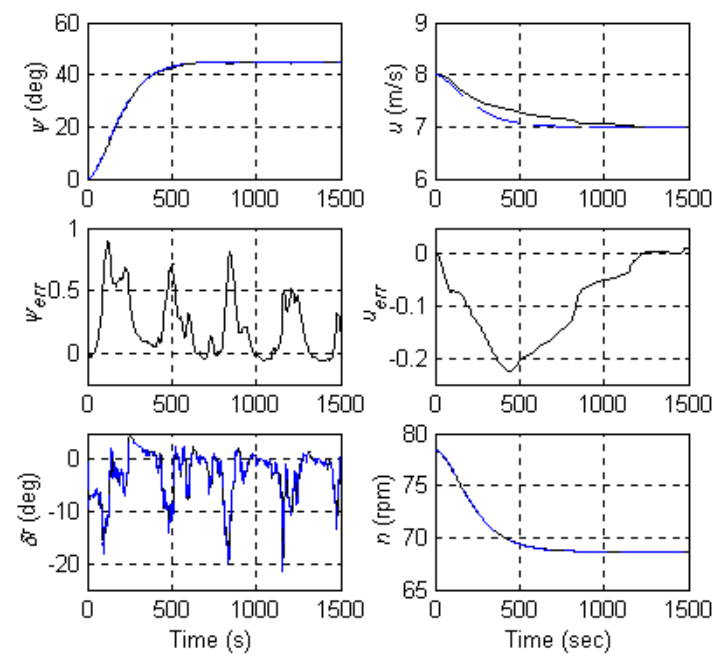

Fig. 7. Sliding mode controller as corrective controller with inverse model as feedforward controller.

By observing the heading error, it can be seen clearly that the heading subsystem has tracked the desired heading with a good level of accuracy. When the rudder deflection response for this manoeuvre is considered, it can be seen that the amplitude limit for this actuator is not exceeded and is in fact well within the $30^{\circ}$ maximum limit. Since the peaks of this response are within the specified rate and magnitude limits, it can be said that the commanded rudder required for this heading manoeuvre is well within the operational envelope of the rudder actuator even in the presence of wave disturbances. This shows that the rudder criterion has been satisfied, i.e. the rudder deflections are kept within the amplitude operating limits.

As in the previous evaluation, the propulsion subsystem is able to provide good surge velocity tracking of $\Delta u= \pm 0.2 \mathrm{~m} / \mathrm{s}$. In addition, it can be seen clearly that the SM controller with inverse model control regulates the shaft velocity tracking with greater accuracy.

\section{CONCLUSION}

The computer simulation results presented in this paper indicate that the use of inverse model feedforward control in conjunction with a corrective feedback controller can provide benefits when compared with a conventional feedback controller. The proposed control structure offers improvements in terms of disturbance rejection while maintaining a high level of tracking accuracy. The inverse model controller 'lightens' the load in terms of control actions and reduces the rudder deflections needed to follow the given trajectories in the presence of significant wave disturbances. This means that further corrective action would be possible if a change in circumstances demanded it.

In addition, it has shown that SM controller is able to provide precise tracking accuracy and offer good disturbance rejection. Furthermore, it has proven that SM controller benefits from the combined control structure and outperformed conventionally pure feedback control methodology.

To summarise, the results from this investigation have indicated that inverse model control working in conjunction with a corrective feedback controller has improved the manoeuvring performance of the oil tanker during course tracking. Consequently, if applied in practice, such a control structure could advantages for future tanker designs.

\section{REFERENCES}

Edwards, C. and Spurgeon, S.K. (1998). Sliding Mode Control: Theory and Application. Taylor and Francis, London.

Fossen, T. I. (1994). Guidance and Control of Ocean Vehicles. John Wiley \& Sons, Chichester.

Healey, A. J. and Marco, D. B. (1992). Slow Speed Flight Control of Autonomous Underwater Vehicles: Experimental Results with NPS AUV II. Proc. of the Second International Offshore and Polar Engineering Conference, San Francisco, 1992, p. 525-532.

Källström, C.G., Åström, K.J., Thorell, N.E., Eriksson, J. and Sten, L. (1979). Adaptive Autopilots for Tankers. Automatica, 15(3), p. 241-254.

Magni, J., Bennani, S. and Terlouw, J. (1997). Robust Flight Control: A Design Challenge. Springer Verlag, Lecture Notes in Control and Information Sciences, 224.

McGookin, E. W., Murray-Smith, D.J., Li, Y. and Fossen, T.I. (2000). The Optimisation of a Tanker Autopilot Control System Using Genetic Algorithms. Transactions of the Institute of Measurement and Control, 22(2), p. 141-178.

Skogestad S. and Postlethwaite, I. (1996). Multivariable feedback control: analysis and design. John Wiley \& Sons, England. 